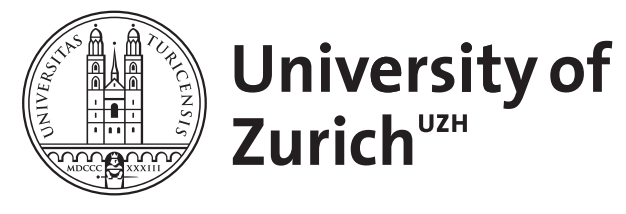

\title{
Resuscitation training for laypersons
}

\author{
Hossli, Georg
}

\begin{abstract}
Emergency medicine is, to a great extent, based on the progress made in clinical anesthesiology and resuscitation. It is only natural for these clinically approved measures, which are routinely used for surgery and anesthesia in recovery rooms and intensive care stations, to be applied outside the hospital, where first aid is required, be this at the scene of an accident or at bedside. An $<$ jats:italic $>$ emergency $</$ jats:italic $>$ exists when "immediate danger to life or danger of serious lasting injury is present, cannot be excluded or has to be expected." This is particularly the case with disturbances of the vital functions, respiration and circulation in polytraumatized patients, for example, with injuries of body cavities, head and cervical spine, hemorrhagic shock, coma or somnolence; or in patients with acute heart insufficiency.
\end{abstract}

DOI: https://doi.org/10.1017/s1049023x00043879

Posted at the Zurich Open Repository and Archive, University of Zurich ZORA URL: https://doi.org/10.5167/uzh-153985

Journal Article

Published Version

Originally published at:

Hossli, Georg (1985). Resuscitation training for laypersons. Prehospital and Disaster Medicine, 1(S1):8385.

DOI: https://doi.org/10.1017/s1049023x00043879 


\section{Resuscitation Training for Laypersons}

\author{
Georg Hossli, MD
}

Emergency medicine is, to a great extent, based on the progress made in clinical anesthesiology and resuscitation. It is only natural for these clinically approved measures, which are routinely used for surgery and anesthesia in recovery rooms and intensive care stations, to be applied outside the hospital, where first aid is required, be this at the scene of an accident or at bedside.

An emergency exists when "immediate danger to life or danger of serious lasting injury is present, cannot be excluded or has to be expected." This is particularly the case with disturbances of the vital functions, respiration and circulation in polytraumatized patients, for example, with injuries of body cavities, head and cervical spine, hemorrhagic shock, coma or somnolence; or in patients with acute heart insufficiency.

An emergency requires a priori or may require later, during transportation:

\section{For respiration:}

- clearing of airways (e.g., suctioning)

- measures to keep airways free /correct positioning and, if necessary, endotracheal intubation)

- artificial respiration

\section{For circulation:}

- control of hemorrhage

- treatment of shock

- cardiac resuscitation

In such situations, the immediate measures that can be expected of a non-medical helper may be life saving or prevent lasting damage to health under certain circumstances. In serious cases, however, extended and appropriate medical help from the trained emergency doctor ladvanced life-support; ALS) is usually of vital importance - provided it arrives in time.

\footnotetext{
From the Department of Anesthesiology, University of Zurich, Cantonspital Zurich, Switzerland.

Presented in June 1981; reviewed by author in 1982
}

On-the-spot first aid is identical with phase one of emergency medicine: the trained non-medical helper, whether chance witness of an accident or the summoned professional helper, as well as the emergency doctor, must pave the way to recovery already on the spot and during transportation: they are, so to speak, the "prolonged arm" of the hospital surgeon, anesthesiologist or emergency internist. The whole structure of prehospital rescue should express that direct life-saving measures are preeminent and their training, equipment and organization have priority. The various phases of prehospital care are hooked together like a chain /"Rescue Chain," an expression created by Ahnefeld). Bearing in mind that a chain is only as strong as its weakest link, it is obvious that only if each link works perfectly will the patient arrive at the hospital in optimal condition.

The objects of rescue and resuscitation are:

- The immediate application of first aid in the form of basic life support by the man in the street that brings the patient into a condition permitting transportation; and

- While continuing with all the necessary measures of upkeep, improvement and control of vital functions, careful transportation to an adequate (and not necessarily the nearest) hospital, which is competent to manage his most serious injury.

Concerning the significance of speed in first aid and transport, the only rational requirements are:

- Performance of immediate life saving measures by the man in the street as soon as possible;

- Arrival of professional helpers and emergency doctor at the scene as soon as possible for the extended life saving measures, and;

- Best possible preparation of the patient for transportation and careful transfer under close supervision and treatment, which means that under these conditions during transport, speed is no longer of primary importance.

There would be little sense in developing in-hospital care for emergency cases with better trained doctors and better diagnostic possibilities, perfected surgery and internal medicine without at the same time improving the services to be rendered beforehand, outside the hospital. According to Ahnefeld's statistical studies, $40 \%$ of emergencies are due to trauma and $60 \%$ to acute life-threatening diseases. The countless possible combinations of injuries, emergencies in internal medicine, obstetrics, pe- 
diatrics, psychiatry and other specialties show how wide the range of emergency medicine is with which the first aiders and other members of the rescue chain may be confronted. The instruction of the helpers for each level will depend on previous knowledge, time allotted, training facilities and available equipment.

In our federalistic state, the standard training program for teaching the basic life saving measures for the man in the street consists of ten lessons. It is actually a training of what must be undertaken in the very first moment at the scene of an accident and how the alarm should be given, then also correct positioning, simple methods of bleeding control and treatment of shock as well as respiration by mouth. This education is compulsory in most parts of the country for pupils of the upper classes aged twelve years and older, and also for driver's license trainees. In the army, it is the essential part of the so-called "self-help" and "comrade's help."

During twenty further lessons, so called "Samaritans" are instructed to use the air-bag-resuscitator and the suction pump and to improvise transportation. For some of them, external cardiac massage is taught in four extra lessons after which participants must pass a test and are given a certificate.

For the non-medical professional helper, such as the paramedical personnel in the rescue services and nurses, more extensive schooling with 50 lessons and at least six half-days of practical work in the emergency admission unit of a large hospital is integrated in their basic instruction. In addition to the curriculum of the first two classes, the care of intubated patients or such with tracheotomy, techniques of intravenous infusions, injection and infusion of drugs and blood substitutes according to doctor's prescription are stressed, as well as assistance to an emergency doctor, for instance during control of bleeding, intubation and cardiac resuscitation.

A doctor working in the rescue services, an "emergency doctor," should not only have special knowledge in the medical field, but also concerning the specific aspects of rescue technology and organization. Anesthetists, surgeons and internists are predestined for such specialized training; in the future, it ought to be included in the curriculum of the medical schools and become part of state examinations all over the world.

Lay helpers present a problem of their own because they do not work professionally with patients. They are often helpless when actually confronted with an emergency situation because their training has not been realistic enough. Psychological stress, fear and surprise as reactions to the uncommon and completely unexpected sight of a seriously injured person, paralyze the helper's ability to make quick decisions or may lead to false steps. As a supplement to first aid introductory courses, training facilities are required, which permit repeated practice in some true-to-life emergency situations. Trainer and trainee should thereby be able to judge the level of knowledge in first aid, especially in immediate life-saving measures.

For this reason, one of my former assistants, Dr. Kurt Reist, initiated the SanArena, an indoor training ground, composed of eight realistic accident situations. The SanArena is supported financially by a bank. It is equipped with special dummies that simulate various difficulties of ventilation and circulation, thus permitting true-to-life training and testing. In three hours, an instructor leads a small group through this "obstacle course" and trainees must handle the following situations: drowning, workshop accident with severe hemorrhage and traumatic amputation, fall from a scaffold, electrocution, burial under wreckage, fire and explosion in a kitchen, sudden fainting and traffic accident on a freeway.

This practical training is followed by a discussion about the errors made. Our experience during the last four years has shown that the SanArena is very suitable for the training of lay helpers. Further development of this training system is planned with improved dummies for the training of professional helpers and especially emergency doctors in such a way that extended life saving measures, such as endotracheal intubation, cricothyrotomy, and emergency puncture of tension pneumothorax, can also be practiced.

At the moment, the relatively expensive SanArena in Zurich is the only one of its kind in Switzerland, where (with a population of 6.5 million/ around 200,000 emergency helpers are educated annually. For this great number of trainees the Swiss Samaritans Association has organized a mobile training unit as a further simple, but effective, training possibility |so-called "NothilfeParcours"|. Such mobile units can be set up in the country, somewhat at a distance from one another. Trainees walk from post to post. Each of these confronts them with a different emergency situation: behavior at the scene of an accident, positioning, basics of respiration, practice of artificial respiration, hemorrhage, giving alarm and general behavior at the scene of a freeway accident, different kinds of accidents (traffic accidents, household accidents, workplace accidents etc.) and general repetition. The program is organized like a competition, with puzzles, games, group work, qualification stickers and so forth. Such a training unit can be set up, for instance, in small towns when the local clubs have an event. Persons who already have basic emergency 
training le.g., for civilians, in the army, in civil defense or in schooll may then participate in such a competition, in small groups of two to five, at the posts of the "NothilfeParcours." These new mobile training units were very well received: in 1980 more than 100,000 emergency helpers took part in 1,022 towns in Switzerland, proving the people's interest in repeated first aid training.

I personally am convinced that the best results in resuscitation training of adult laypersons can be achieved by emphasizing the principle of voluntariness and by appealing to the common sense of everyone.

\section{Emergency Medicine in the United Kingdom-How It Is Taught}

\author{
Andrew K. Marsden, MB, ChB, FRCSEd
}

The staff of accident and emergency departments in the United Kingdom are realizing their increasing role in teaching the elements of emergency care. This paper highlights some recent developments which have influenced the way in which emergency medicine is taught.

First Aid instruction aims to impart the universally agreed and accepted principles to a wide ranging audience. Traditionally these principles have been embodied in the combined manual of the St. John Ambulance Service, St. Andrew's Ambulance Service and the British Red Cross Society. The 1974 Health and Safety at Work Act has laid down revised standards for Industrial First Aid and 1981 saw the introduction of a First Aid at Work Act which incorporates stringent requirements in terms of the level and performance of First Aid activity within any place of employment. Educational institutions and even hospitals have no immunity from these regulations. Now First Aid will be linked with Occupational Medicine, and instruc-

From the Pinderfields General Hospital Wakefield, West Yorkshire, United Kingdom

Presented in 1981; reviewed by author in 1982 tors to industry will need to be approved by the Health and Safety Executive-a controlling body with statutory powers. The voluntary aid societies are reassessing their role with a view to an extended five day course based on a new First Aid Manual for Industry, leading to a Certificate of Competence in Occupational First Aid. Initiative in this respect has already been shown by some medical manufacturing companies. The equipment firm of AMBU International UK has, for some years now, been presenting intensive courses in First Aid and resuscitation for industry.

Training of the general public in cardiopulmonary resuscitation (CPR) Basic Life Support has gained momentum slowly in Great Britain. It is with traditional British conservatism that we lag behind the Americans and Scandinavians in this field. The progress that has been made is due to the efforts of schools and youth movements. The British Association for Immediate Care aims to develop this activity and to extend the progress achieved by Resuscitation Training Schemes in Brighton, Avon and Gloucester. The accreditation of trainers and testing of trainees demands standardization to a national norm. Simulator equipment should be able to indicate and record acceptable performance in achieving the prescribed standard.

\section{Ambulance Staff}

The incorporation into the Health.Service in 1974 of the local authority for ambulance services has permitted the embodiment of national standards for basic ambulance training. A basic trained ambulanceman will have attended a six week course; received driving instruction; benefited from in-hospital exposure; and been practically supervised on the road by his training officer for at least 12 months. He is then awarded the Millar Proficiency Certificate. Further training by refresher courses ensures regular updating in the practical skills.

Advanced Ambulance (paramedic) Training has occurred sporadically, but always with vigor and enthusiasm. The application of advanced techniques must be based on the firm knowledge of facts and their application to disease processes. We see four levels of training:

Phase One is a self-learning course in the basic sciences. Phase Two, ambulance aid, is acquired by practical experience in the field. Phase Three comprises small group teaching in hospitals of such subjects as ECG interpretation and principles of drug administration. In Phase Four the student acquires advanced practical skills 